\title{
MULTIPLIERS AND LOCAL SPECTRAL THEORY
}

\author{
KJELD BAGGER LAURSEN \\ Matematisk Institut, Københavns Universitet \\ Universitetsparken 5, DK-2100 København Ø, Denmark
}

1. Brief introduction to multipliers. Multipliers are operators on Banach algebras with immediate appeal. Some of this appeal comes from the very properties that define them, emulating, as they do, the basic operation of "multiplication" in an algebra. Some of it has to do with the structure results they naturally give rise to. By explaining the situation in group algebras I can probably make it clear what I mean:

The context in which we shall be working will be that of a commutative Banach algebra $A$. Any element $a \in A$ gives rise to a multiplication operator $T_{a}: A \rightarrow A$, defined by $T_{a} b:=a b$ for all $b \in A$. Clearly each such $T_{a}$ is a continuous linear operator. It is also obvious that because $A$ is commutative $T_{a}$ will commute with every other multiplication operator. Might this characterize multiplication operators? In other words, if $T$ is a linear operator on $A$ for which $T(a b)=T(a) b$ for all $a, b \in A$, will $T$ itself have to be a multiplication operator? If $A$ has a unit 1 , the answer is easily yes, because $T a=T(1 a)=T(1) a$, so that $T=T_{T(1)}$. However, as Wendel $[\mathrm{W}]$ and Helson $[\mathrm{H}]$ independently showed, around 1952 , if we turn to the group algebra $A:=L^{1}(G)$, where $G$ is a locally compact abelian group, the requirement on our sought-after operators, that of commuting with all multiplication operators, leads to a much larger class of operators: these operators turn out to coincide with the set of convolution operators $T_{\mu}$, defined by the formula $T_{\mu} a:=\mu * a$ for all $a \in L^{1}(G)$, as $\mu$ ranges over the set of complex Borel measures on $G$.

1991 Mathematics Subject Classification: 47C05, 47B40, 43A22.

The paper is in final form and no version of it will be published elsewhere.

A portion of the material of this paper was presented to the Banach Centennial Semester in May 1992.

In the preparation of the final version I have benefited greatly from many comments and suggestions from Michael M. Neumann and Jaroslav Zemánek. I want to express my appreciation of this help. 
To specify what we take for granted as well as what notation to use, we begin with a telegrammatic introduction to the theory of multipliers on commutative Banach algebras $[\mathrm{L}]$. Let $A$ be a commutative Banach algebra. The basics of the theory of multipliers is easily developed on assumptions weaker than the one we shall generally make here (cf. [L]). To avoid cluttering the exposition, however, we shall make the blanket assumption that $A$ be semisimple: we assume the Gelfand transform $a \rightarrow a^{\wedge}$ to be an injection (i.e. one-to-one).

Let $\Phi_{A}$ denote the space of multiplicative linear functionals on $A$. Usually we shall equip $\Phi_{A}$ with the topology of pointwise convergence: a net $\left\{\phi_{\alpha}\right\}$ in $\Phi_{A}$ converges to $\phi \in \Phi_{A}$ if $\phi_{\alpha}(a)$ converges to $\phi(a)$ for all $a \in A$; this way $\Phi_{A}$ becomes a locally compact Hausdorff space. The Gelfand transform $a^{\wedge}$ of an element $a \in A$ is the continuous complex-valued function on $\Phi_{A}$, vanishing at infinity, which is defined by $a^{\wedge}(\phi):=\phi(a)$ for every $\phi \in \Phi_{A}$.

One common definition of the set of multipliers $M(A)[\mathrm{L}]$ specifies that $M(A)$ consists of all operators $T: A \rightarrow A$ for which $a(T b)=(T a) b$ for all $a, b \in A$. For a semisimple algebra this is immediately seen to be equivalent with the one mentioned earlier, so examples of multipliers have already been given. An easy argument [L], involving the closed graph theorem, the definition of multiplier and the semisimplicity of $A$ also ensures that all multipliers are necessarily both continuous and linear, so $M(A)$ becomes a closed commutative subalgebra of $\mathcal{L}(A)$, the bounded linear operators on $A$, when $M(A)$ and $\mathcal{L}(A)$ are normed by the usual operator norm. As we have already pointed out, $A$ can be viewed as a subalgebra, in fact as an ideal, of $M(A)$, but the given norm on $A$ may well differ from the operator norm $[\mathrm{L}]$.

Every $T \in M(A)$ defines a bounded continuous complex-valued function $T^{\wedge}$ on $\Phi_{A}$, which is given by taking Gelfand transforms: $(T a)^{\wedge}=T^{\wedge} a^{\wedge}$, for any $a \in A$. This means that for every $\phi \in \Phi_{A}$ there is a complex number $T^{\wedge}(\phi)$ for which $T^{\wedge}(\phi) a^{\wedge}(\phi)=(T a)^{\wedge}(\phi)$ for every $a \in A$. Since $\Phi_{A}$ is a subset of the dual space $A^{\prime}$, we might note in passing that $T^{\wedge}$ is simply the restriction of the dual $\operatorname{map} T^{\prime}: A^{\prime} \rightarrow A^{\prime}$ to $\Phi_{A}:\left(T^{\prime} \mid \Phi_{A}\right)(\phi)(a)=T^{\wedge}(\phi) a^{\wedge}(\phi)$ for every $\phi \in \Phi_{A}$ and $a \in A$.

Naturally, if $T^{\wedge}$ is to provide us with much information about $T$, we must have a close connection between them. This is achieved here by assuming $A$ to be semisimple: in that case the correspondence $T \leftrightarrow T^{\wedge}$ is injective.

Note that since $M(A)$ is a commutative Banach algebra with a maximal ideal space $\Phi_{M(A)}$ we may also talk about the Gelfand transform of $T$ as an element of $M(A)$. We shall make occasional use of this and when we do the notation will also be $T^{\wedge}$, with the domain of definition specified as $\Phi_{M(A)}$. There is no serious abuse of notation in this because with the description of $\Phi_{A}$ as a subset of $\Phi_{M(A)}$ that we now give, $T^{\wedge}$, defined on $\Phi_{A}$, is simply the restriction of the "ordinary" Gelfand transform $T^{\wedge}$ on $\Phi_{M(A)}$.

Semisimplicity of $A$ tells us that $\Phi_{A}$ separates the points of $M(A)^{\wedge}\left(\right.$ if $T^{\wedge}=S^{\wedge}$ on $\Phi_{A}$ then $(T a)^{\wedge}=(S a)^{\wedge}$ for every $a \in A$, hence $T=S$, by semisimplicity), 
so we may view $\Phi_{A}$ as a subset of $\Phi_{M(A)}$ : each $\phi \in \Phi_{A}$ determines an element of $\Phi_{M(A)}$ by the assignment $\phi(T):=T^{\wedge}(\phi)$. To obtain the rest of $\Phi_{M(A)}$ from $\Phi_{A}$ we throw in all elements of $\Phi_{M(A)}$ that vanish on $A$, i.e. the hull of $A \subseteq M(A)$. This gives us a description of $\Phi_{M(A)}$ as

$$
\Phi_{M(A)}=\Phi_{A} \cup \operatorname{hull}_{\Phi_{M(A)}}(A) .
$$

This description covers not just the set theoretic situation, but also the topological one: the weak ${ }^{*}$ topology of $\Phi_{A}$ is the restriction of the weak ${ }^{*}$ topology on $\Phi_{M(A)}[\mathrm{L}]$.

The elements of $A$ and of $M(A)$ may be thought of as continuous functions on $\Phi_{M(A)}$, with the former vanishing at infinity as functions on $\Phi_{A}$ and on all of hull $_{\Phi_{M(A)}}(A)$ as functions on $\Phi_{M(A)}$. We shall have occasion to study two ideals of $M(A)$, namely

$$
M_{0}(A):=\left\{T \in M(A) \mid T^{\wedge} \text { vanishes at infinity on } \Phi_{A}\right\},
$$

and

$$
M_{00}(A):=\left\{T \in M(A) \mid T^{\wedge} \text { vanishes on } \operatorname{hull}_{\Phi_{M(A)}}(A)\right\} .
$$

For these subsets of $M(A)$ much more detailed results are known; this is hardly surprising, since the asymptotic behavior of their elements is known. Right off, this chain of inclusions is clear:

$$
A \subseteq M_{00}(A) \subseteq M_{0}(A) \subseteq M(A) .
$$

These inclusions may well all be strict; indeed they all are, as it is exhibited in [GMcG], whenever $A:=L^{1}(G)$ for any non-discrete locally compact abelian group $G$.

2. Brief introduction to local spectral theory. In 1968 Colojoară and Foiaş published their pioneering study of local spectral theory $[\mathrm{CF}]$. To appreciate what they did, and some of what has happened since, it is reasonable first to come to grips with what "decomposability" means: if for a given continuous linear operator $T$ on a Banach space $X$, one knows that it possesses some of the pleasant properties that a normal operator on a Hilbert space has, one would seem to know quite a bit about this operator. It is this admittedly vague guiding principle that lies behind much of the development of local spectral theory. Specifically, let us agree to call $T \in \mathcal{L}(X)$ decomposable if any open cover $\{U, V\}$ of the complex plane $\mathbb{C}$ allows a splitting of $X$ into the algebraic sum (ordinarily not direct) $X=Y+Z$ of two closed $T$-invariant subspaces $Y$ and $Z$ on which the restriction has "smaller" spectra: $\sigma(T \mid Y) \subseteq U$ and $\sigma(T \mid Z) \subseteq V$.

It is not just the decomposition of spectra of decomposable operators that justifies the term "local" spectral theory; the theory goes well beyond this level of generality, based, as it is, on the notion of local spectrum.

Given $T \in \mathcal{L}(X)$ and $x \in X$, the resolvent function $(T-\lambda)^{-1}$, defined for all $\lambda \in \mathbb{C} \backslash \sigma(T)$, i.e. for all complex $\lambda$ outside the spectrum $\sigma(T)$ of $T$, provides us 
with an analytic solution of the equation

$$
(T-\lambda) x(\lambda)=x,
$$

namely $x(\lambda):=(T-\lambda)^{-1} x$, for every $\lambda \in \mathbb{C} \backslash \sigma(T)$. This function may well have analytic extensions, at least for certain $x \in X$. A normal operator $N \in \mathcal{L}(\mathcal{H})$ on the Hilbert space $\mathcal{H}$ will exemplify how this may be: if the spectral projections of $N$ are denoted by $E(\Delta), \Delta$ a Borel set, and if $h \in E(\Delta) \mathcal{H}$ for some such $\Delta$, then $(N \mid E(\Delta) \mathcal{H})-\lambda$ is invertible for all $\lambda \in \mathbb{C} \backslash(\sigma(N) \cap \Delta)$, and so in this case the equation $(N-\lambda) h(\lambda)=h$ has an analytic solution on $\mathbb{C} \backslash(\sigma(N) \cap \Delta)$.

Returning to the general situation of $T \in \mathcal{L}(X)$ and $x \in X$, the union of all open subsets of $\mathbb{C}$ on which $(T-\lambda) x(\lambda)=x$ has an analytic solution is called the local resolvent of $T$ at $x$ and is denoted by $\varrho_{T}(x)$.

It should be noted right away that such analytic solutions are not necessarily uniquely determined. That depends on whether or not the equation $(T-$ $\lambda) x(\lambda)=0$ has non-trivial analytic solutions on any open subset of the plane. If our $T \in \mathcal{L}(X)$ yields only the zero solution of this equation, we say that $T$ has the single-valued extension property $(S V E P)$. Any decomposable operator has SVEP [CF, Corollary 2.1.4]. The left shift $L$ on $\ell^{2}(\mathbb{N})$, say, does not: $(L-$ $\lambda)\left(\lambda, \lambda^{2}, \lambda^{3}, \ldots\right)=0$ for all $\lambda \in \mathbb{C},|\lambda|<1$, and the function $\lambda \rightarrow\left(\lambda, \lambda^{2}, \lambda^{3}, \ldots\right)$ is an analytic function from the open unit disc into $\ell^{2}(\mathbb{N})$.

The complement $\sigma_{T}(x):=\mathbb{C} \backslash \varrho_{T}(x)$ is the local spectrum of $T$ at $x$. This set is, largely, the main technical object of study in local spectral theory. If $F \subseteq \mathbb{C}$ is a given closed set then the set

$$
X_{T}(F):=\left\{x \in X \mid \sigma_{T}(x) \subseteq F\right\}
$$

is called the analytic spectral subspace (associated with $T$ and $F$ ). The word "subspace" is clearly not misused here. A comprehensive development of these concepts may also be found in [V2].

Much local spectral theory has been developed with the standing assumption that the operator in question have SVEP. To illustrate that this is often not at all relevant we include here some descriptions of $X_{T}(F)$ for certain $F \subseteq \mathbb{C}$. These results will also establish a connection to Kato's perturbation theory $[\mathrm{K}]$. There are related results in the work of Mbekhta, e.g. [M].

Consider the set that we shall call the Kato resolvent, a set containing the ordinary resolvent, and defined for $T \in \mathcal{L}(X)$ by

$$
\varrho_{K}(T):=\left\{\lambda \in \mathbb{C} \mid(T-\lambda) X \text { is closed and } \operatorname{ker}(T-\lambda) \subseteq \bigcap_{n \in \mathbb{N}}(T-\lambda)^{n} X\right\} .
$$

The Kato resolvent is open [K, Theorem 3, p. 297] and may well contain points of the spectrum $\sigma(T)$, as suggested, for instance, by the observation that if $\lambda$ lies outside the approximate point spectrum $\sigma_{\text {ap }}(T)$, then $T-\lambda$ is bounded below (cf. e.g. [C, Proposition VII.6.4]), and hence $\mathbb{C} \backslash \sigma_{\text {ap }}(T) \subseteq \varrho_{K}(T)$ (this containment may be improved upon [LN2]). 
To make the connection to local spectral theory it is convenient to recall that for a subset $C \subseteq \mathbb{C}$ the algebraic spectral subspace $E_{T}(C)$ is defined as the algebraic sum of all subspaces of $X$ on which all the restrictions of $T-\lambda, \lambda \in \mathbb{C} \backslash C$, are surjective. Thus $E_{T}(C)$ is the largest subspace of $X$ with this surjectivity property. Clearly, from the definition, $E_{T}(C) \subseteq \bigcap_{\lambda \in \mathbb{C} \backslash C} \bigcap_{n \in \mathbb{N}}(T-\lambda)^{n} X$. Also, it follows from elementary local spectral theory that $X_{T}(F) \subseteq E_{T}(F)$, for any closed $F \subset \mathbb{C}$.

We then have the following description of situations in which these containments are equalities.

Theorem 1. Let $T \in \mathcal{L}(X)$ and $\lambda \in \mathbb{C}$. If $\operatorname{ker}(T-\lambda) \subseteq \bigcap_{n \in \mathbb{N}}(T-\lambda)^{n} X$ then $E_{T}(\mathbb{C} \backslash\{\lambda\})=\bigcap_{n \in \mathbb{N}}(T-\lambda)^{n} X$. If, moreover, $\lambda \in \varrho_{K}(T)$, then $E_{T}(\mathbb{C} \backslash\{\lambda\})$ is closed; in particular, for every subset $G \subseteq \varrho_{K}(T), E_{T}(\sigma(T) \backslash G)$ is closed, hence, if $G$ is open, $E_{T}(\sigma(T) \backslash G)=X_{T}(\sigma(T) \backslash G)$.

Pro of. The inclusion $E_{T}(\mathbb{C} \backslash\{\lambda\}) \subseteq \bigcap_{n \in \mathbb{N}}(T-\lambda)^{n} X$ always holds. For the reverse inclusion it suffices to show that $\bigcap_{n \in \mathbb{N}}(T-\lambda)^{n} X \subseteq(T-\lambda) \bigcap_{n \in \mathbb{N}}(T-\lambda)^{n} X$ and this is an easy consequence of the assumption $\operatorname{ker}(T-\lambda) \subseteq \bigcap_{n \in \mathbb{N}}(T-\lambda)^{n} X$. Now, if $\lambda \in \varrho_{K}(T)$ then the closedness of $\bigcap_{n \in \mathbb{N}}(T-\lambda)^{n} X$ is shown in [S, proof of Satz 4, p. 487]. The closedness of $E_{T}(\sigma(T) \backslash G)$ follows from this, since $E_{T}(\sigma(T) \backslash G)=\bigcap_{\lambda \in G} E_{T}(\mathbb{C} \backslash\{\lambda\})[\mathrm{PV}]$. So, as a consequence of [LV1, Proposition 10], $E_{T}(\sigma(T) \backslash G)=X_{T}(\sigma(T) \backslash G)$ for every open subset $G \subseteq \varrho_{K}(T)$.

There is more: the spaces $\bigcap_{n \in \mathbb{N}}(T-\lambda)^{n} X$ are known to be constant as $\lambda$ ranges over a connected component $G$ of $\varrho_{K}(T)[\mathrm{F}]$, so we may of course draw the same conclusion for $X_{T}(\mathbb{C} \backslash\{\lambda\})$. This constant value is also $X_{T}(\sigma(T) \backslash G)$, because $X_{T}(\sigma(T) \backslash G)=\bigcap_{\mu \in G} X_{T}(\mathbb{C} \backslash\{\mu\})$. Moreover, as we shall now see, $\bigcap_{\mu \in G}(T-\mu) X=\bigcap_{n \in \mathbb{N}}(T-\lambda)^{n} X$, i.e. we only have to consider the first power of $T-\mu$ as $\mu$ ranges over $G$. This leads to the following explicit formulas. For a related result see Theorem 8 .

THEOREM 2. For $\lambda$ ranging through a connected component $G$ of $\varrho_{K}(T)$ the spaces $X_{T}(\mathbb{C} \backslash\{\lambda\})$ are constant; in fact, for any $\lambda \in G$,

$$
X_{T}(\mathbb{C} \backslash\{\lambda\})=X_{T}(\sigma(T) \backslash G)=\bigcap_{\mu \in G}(T-\mu) X=\bigcap_{n \in \mathbb{N}}(T-\lambda)^{n} X .
$$

Proof. With no loss of generality take $\lambda=0$, so that $G$ is a neighborhood of 0 . Theorem 1 shows that the extremes in the above string of spaces are equal. It remains to establish that $\bigcap_{n \in \mathbb{N}} T^{n} X \supseteq \bigcap_{\mu \in G}(T-\mu) X$. An argument as employed in proving [SW, Proposition 2.7] will do this: because $0 \in \varrho_{K}(T)$ the map

$$
S: T X \rightarrow X / \bigcap_{n \in \mathbb{N}} T^{n} X, \quad T x \rightarrow x / \bigcap_{n \in \mathbb{N}} T^{n} X,
$$

is well defined; as in [SW], $S$ is continuous and $\operatorname{ker} S=\bigcap_{n \in \mathbb{N}} T^{n} X$. Let $y \in$ $\bigcap_{\mu \in G}(T-\mu) X$. Then $y=(T-\mu) x_{\mu}$, i.e. $T x_{\mu}=y+\mu x_{\mu}$ for every $\mu \in G$, and 
hence

$$
x_{\mu} / \bigcap_{n \in \mathbb{N}} T^{n} X=S T x_{\mu}=S\left(y+\mu\left(x_{\mu}+z\right)\right),
$$

for any choice of $z \in \bigcap_{n \in \mathbb{N}} T^{n} X$, from which we obtain the estimate

$$
\left\|x_{\mu} / \bigcap_{n \in \mathbb{N}} T^{n} X\right\| \leq\|S\|\left(\|y\|+|\mu|\left\|x_{\mu} / \bigcap_{n \in \mathbb{N}} T^{n} X\right\|\right) .
$$

For sufficiently small $\mu$ we conclude that

$$
\left\|x_{\mu} / \bigcap_{n \in \mathbb{N}} T^{n} X\right\| \leq 2\|S\| \cdot\|y\| .
$$

It follows from $T x_{\mu}=y+\mu x_{\mu}$ that

$$
\left\|\left(T x_{\mu}-y\right) / \bigcap_{n \in \mathbb{N}} T^{n} X\right\|=|\mu|\left\|x_{\mu} / \bigcap_{n \in \mathbb{N}} T^{n} X\right\| \rightarrow 0 \quad \text { as } \mu \rightarrow 0 .
$$

As $\bigcap_{n \in \mathbb{N}} T^{n} X \subseteq T X$ we conclude that $y \in T X$. The equation $T x_{\mu}=y+\mu x_{\mu}$ then shows that $x_{\mu} \in T X$. Repetition of the last two lines yields the conclusion that $y \in T^{2} X$, which is closed by [S, Satz 4]. By induction we see that $y \in \bigcap_{n \in \mathbb{N}} T^{n} X$.

EXAMPLE. Let $T$ be an isometry on the Banach space $X$. If $T$ is non-invertible then $\sigma(T)=\mathbb{D}$, the closed unit disc. It is easy to see that $\varrho_{K}(T) \cap \sigma(T)=\mathbb{D}^{\circ}$, the open unit disc. Thus, from Theorems 1 and 2 we conclude that for every $\lambda \in \mathbb{D}^{\circ}$,

$$
E_{T}(\mathbb{C} \backslash\{\lambda\})=X_{T}(\mathbb{T})=E_{T}(\mathbb{T})=\bigcap_{\mu \in \mathbb{D}^{\circ}}(T-\mu) X=\bigcap_{n \in \mathbb{N}} T^{n} X .
$$

Here $\mathbb{T}$ is the unit circle. Of course, if $T$ is an invertible isometry, the above string of equalities also holds because then $\sigma(T) \subseteq \mathbb{T}$, and so the spaces all equal $X$. Some related remarks may be found in [LV2].

3. The two shall meet. The spectral theorem for normal operators on a Hilbert space tells us that normal operators are decomposable. Of course there are many other interesting classes of examples. Here, as announced, we shall concentrate on the decomposability properties that multipliers might possess.

Colojoară and Foiaş looked into this: their monograph contains the beginning of a study of decomposability properties of multipliers on regular algebras. Recall that a commutative semisimple Banach algebra $A$ is regular if, as an algebra of functions on its maximal ideal space $\Phi_{A}$, the algebra $A^{\wedge}$ is large enough that any point $\phi$ of $\Phi_{A}$ and any closed set $F \subseteq \Phi_{A}$ not containing $\phi$ may be separated by an element of $A^{\wedge}$; "separated" means that there is $a^{\wedge} \in A^{\wedge}$ which vanishes everywhere on $F$, but not at $\phi$. They showed that if the commutative Banach algebra $A$ is semisimple and regular then any multiplication operator $T_{a}$ on $A$ will be decomposable. 
This immediately brings to mind the following two natural questions:

1) This result is proved for a multiplication operator, not an arbitrary multiplier. Might multipliers all be decomposable? And in the same spirit:

2) How significant is the assumption that $A$ be regular?

Take the second question first. Frunză [Fr] showed that the assumption is very reasonable, indeed: he proved that decomposability of all of the multiplication operators on a commutative semisimple Banach algebra is equivalent to regularity.

Thus, whenever our algebra $A$ is non-regular there will be non-decomposable multiplication operators on it. So what distinguishes the decomposable multiplication operators from the others? Neumann $[\mathrm{N}]$ found the answer. To appreciate it we need the following.

The maximal ideal space $\Phi_{A}$ carries two naturally defined topologies, the Gelfand topology and another coarser one, the hull-kernel topology. The latter topology is specified by its closed sets: a subset $F \subseteq \Phi_{A}$ is $h k$-closed if

$$
F=\left\{\phi \in \Phi_{A} \mid \phi(a)=0 \text { for every } a \in A \text { for which } a^{\wedge}(F)=\{0\}\right\} .
$$

It is a classical result, due to Shilov (cf. $[\mathrm{BD}]$ ), that a commutative semisimple Banach algebra $A$ is regular precisely when the Gelfand topology and the hullkernel topology coincide.

Neumann showed that decomposability of $T_{a}$ is equivalent with $a^{\wedge}$ being hullkernel continuous.

TheOREM $3[\mathrm{~N}$, Theorem 1.2]. If $A$ is a commutative semisimple Banach algebra with maximal ideal space $\Phi_{A}$ and if $a \in A$ is given then the multiplication operator $T_{a}: A \rightarrow A$ is decomposable if and only if $a^{\wedge}: \Phi_{A} \rightarrow \mathbb{C}$ is continuous in the hull-kernel topology.

Note how Frunză's result follows immediately from this: if all the functions $a^{\wedge}$ are hull-kernel continuous on $\Phi_{A}$ then the hull-kernel topology on $\Phi_{A}$ must be a Hausdorff topology, hence it must coincide with the Gelfand topology. This means that $A$ is regular [BD, Theorem II.23.8].

To what extent the hull-kernel continuity of $T^{\wedge}$ is a way of characterizing decomposability (on $A$ ) is not yet fully understood, because there are several spaces on which $T$ may be decomposable: $A$ or $M(A)$, and there are several different maximal ideal spaces. The hull-kernel continuity of $T^{\wedge} \mid \Phi_{A}$ is necessary, as we shall now see. It is not sufficient (cf. below).

TheOREM 4 [LN1, Theorem 2.3]. Let A be a commutative semisimple Banach algebra and $T \in M(A)$ be any multiplier. Then (a) implies (b) and (b) implies (c), where

(a) $T^{\wedge}$ is hull-kernel continuous on $\Phi_{M(A)}$.

(b) $T: A \rightarrow A$ is decomposable.

(c) $T^{\wedge} \mid \Phi_{A}$ is hull-kernel continuous on $\Phi_{A}$. 
If $T \in M_{0}(A)$ then (a) and (b) are equivalent; these conditions are fulfilled by $T \in M_{0}(A)$ if and only if $T \in M_{00}(A)$ and satisfies $(\mathrm{c})$.

If $A$ is regular then hull-kernel continuity of $T^{\wedge} \mid \Phi_{A}$ is automatic. As will be seen later, when we have discussed the concept of natural spectrum (cf. the comments after Theorem 8), there are indeed non-decomposable multipliers, even on regular algebras, and hence (c) is strictly weaker than the other two conditions.

The last claim of Theorem 4 tells us that a multiplier $T \in M_{0}(A)$ is decomposable precisely when it lies in $M_{00}(A)$ and is hull-kernel continuous on $\Phi_{A}$. One might wonder whether these latter two conditions are independent, i.e. whether it is possible for a multiplier $T \in M_{0}(A)$ to be hull-kernel continuous on $\Phi_{A}$ without being an element of $M_{00}(A)$ ? The answer is yes. Again an example is more readily found if we tie this question in with the issue of natural spectrum [LN1], [Z] (cf. Section 4 below).

Sets of continuous functions, such as those referred to in Theorem 4(a) or (c), have very pleasant algebraic properties, being algebras, i.e. stable under addition and multiplication. On the other hand, the set of decomposable operators is in general a rather unstructured set: not every operator on Hilbert space can be decomposable (take a non-invertible isometry, for instance: an isometry will necessarily have complex numbers of unit modulus in its spectrum. This makes it impossible to split off subsets of the interior of the unit disc in $\mathbb{C}$ ); but every operator, as the sum of its real and its imaginary part, will be the sum of two normal, hence decomposable operators. This shows that the decomposable operators do not form an additive set. However, in the case of decomposable commuting operators there is considerable evidence that the sum and product of two such will again be decomposable (cf. e.g. [Ap], [E]), but the issue is still not completely settled. For multipliers, however, results such as Theorem 4 indicate that the set $D M(A)$ of multipliers which are decomposable operators on $A$ may well be rather well-behaved, algebraically: for instance, Theorem 4 says that $M_{0}(A) \cap D M(A)$ is an algebra. Theorem 6 will tell us more about this set, but probably the best general result along these lines is due to Albrecht; in [A1, Theorem 2.6] he proves the following, for any Banach algebra. We formulate at our level of generality.

TheOREm 5. If A has a bounded approximate identity then $D M(A)$ is a closed subalgebra of $M(A)$.

Albrecht's proof is based on the theory of functions of several complex variables. It is quite involved and it seems plausible that in the commutative case an easier argument might be found, based, for instance, on considerations of hullkernel continuity. There is a very nice discussion of related matters in Section 3 of $[\mathrm{MN}]$.

It is not surprising that we get much stronger conclusions if we assume that $T$ is an element of $M_{0}(A)$, or even of $M_{00}(A)$. Theorem 4 was one such example. Here are two more. Another one will follow later (Theorem 11). 
TheOREM 6 [LN1, Theorem 2.10(c)]. The algebra $M_{0}(A) \cap D M(A)$ coincides with the set of elements of $M_{0}(A)$ that are decomposable multiplication operators on $M_{0}(A)$. This set is always a subset of $M_{00}(A)$ and coincides with it when $A$ is regular.

Even more definitive information becomes available to us if we make additional topological assumptions on the maximal ideal space $\Phi_{A}$. A topological space is scattered if every non-empty compact subset of it contains an isolated point. Obvious examples are discrete spaces. For commutative group algebras $A:=$ $L^{1}(G)$, where $\Phi_{A}$ may be identified with the dual group $\Gamma$, any compact abelian group $G$ will give us an example of a Banach algebra $A$ with scattered $\Phi_{A}$.

TheOrem 7 [LN1, Theorem 3.1]. If $A$ is a commutative semisimple Banach algebra with scattered maximal ideal space $\Phi_{A}$ and if $T \in M_{0}(A)$ then these statements are all equivalent:

(a) $T^{\wedge}$ is hull-kernel continuous on $\Phi_{M(A)}$.

(b) $T: A \rightarrow A$ is decomposable.

(c) $L_{T}: M_{0}(A) \rightarrow M_{0}(A)$ is decomposable.

(d) $T$ has countable spectrum.

(e) $T \in M_{00}(A)$.

Any multiplier, decomposable or not, will have SVEP, if the algebra $A$ is semisimple. This is clear because the equation $(T-\lambda) x(\lambda)=0$, with $\lambda$ ranging through some open set $G \subseteq \mathbb{C}$, will Gelfand-transform to $\left(T^{\wedge}(\phi)-\lambda\right) x(\lambda)^{\wedge}(\phi)=$ 0 for each $\phi \in \Phi_{A}$. For each $\phi \in \Phi_{A} x(\lambda)^{\wedge}(\phi)=0$ on $G \backslash\left\{T^{\wedge}(\phi)\right\}$, hence by continuity $x(\lambda)^{\wedge}(\phi)=0$ for all $\lambda \in G$. The semisimplicity then shows that $x(\lambda)=0$ on $G$. Knowing now that the equation $(T-\lambda) x(\lambda)=x$ will have a unique analytic solution on the set $\varrho_{T}(x)$, we may turn our attention to the task of describing the analytic spectral subspaces $A_{T}(F)$ for any multiplier $T$. It is quite straightforward to see that for every closed set $F \subseteq \mathbb{C}$,

$$
A_{T}(F) \subseteq Z_{T}(F):=\left\{a \in A \mid \operatorname{supp} x^{\wedge} \subseteq T^{-1}(F)\right\},
$$

where $\operatorname{supp} x^{\wedge}:=\left\{\phi \in \Phi_{A} \mid x^{\wedge}(\phi) \neq 0\right\}^{-}$, the closure taken in the Gelfand topology. This is just another way of saying that for every $a \in A$, the set $T^{\wedge}\left(\operatorname{supp} a^{\wedge}\right)^{-}$ is a subset of the local spectrum $\sigma_{T}(a)$.

Colojoară and Foiaş [CF, Chapter 6.2] showed that if $A$ is regular and if $T:=T_{b}$ is a multiplication operator then there is equality $\sigma_{T}(a)=T^{\wedge}\left(\operatorname{supp} a^{\wedge}\right)^{-}$ for every $a \in A$. It would be entirely natural to repeat the above two questions 1 ) and 2 ) in this context. To use a descriptive phrase, if a multiplier $T$ has the property that $\sigma_{T}(a)=T^{\wedge}\left(\operatorname{supp} a^{\wedge}\right)^{-}$for every $a \in A$, then we shall say that $T$ has natural local spectra.

It is interesting to note that the inclusion $A_{T}(F) \subseteq Z_{T}(F)$ can be augmented to include a middle term, namely $\bigcap_{\lambda \in \mathbb{C} \backslash F}(T-\lambda) A$, which resembles what we 
have seen in connection with Theorem 2. Thus there is this nest of spaces:

$$
A_{T}(F) \subseteq \bigcap_{\lambda \in \mathbb{C} \backslash F}(T-\lambda) A \subseteq Z_{T}(F) ;
$$

this means, in particular, that when the outer terms coincide, we get a description of $A_{T}(F)$ like what was obtained for the Kato resolvent. We cite but a special case:

THEOREM 8 [LN1, Theorem 2.4]. If $T \in M(A)$ and $T^{\wedge}$ is hull-kernel continuous on $\Phi_{M(A)}$ then for every closed subset $F \subseteq \mathbb{C}$,

$$
A_{T}(F)=\bigcap_{\lambda \in \mathbb{C} \backslash F}(T-\lambda) A=Z_{T}(F) .
$$

Somewhat different descriptions, but still rather closely related to the one given here, of the spectral subspaces of a decomposable multiplier may be found in [A2, Theorem 4.5] and [E, Lemma 2.1].

4. Natural spectrum and natural local spectrum. One of the pleasant basic facts for any commutative Banach algebra $A$ is that the spectrum $\sigma\left(T_{a}\right)$ for any element $a \in A$ is computable as the closure of the range $a^{\wedge}\left(\Phi_{A}\right)^{-}$of the Gelfand transform. As a replay of our theme we might wonder whether this is true for all multipliers, and not just for multiplication operators. It is clear that for any $T \in M(A)$ the inclusion $\sigma(T) \supseteq T^{\wedge}\left(\Phi_{A}\right)^{-}$always holds. If $T$ has the property that $\sigma(T)=T^{\wedge}\left(\Phi_{A}\right)^{-}$then we shall say that $T$ has natural spectrum. Thus, every multiplication operator $T_{a}, a \in A$, has natural spectrum. Moreover, since $\sigma(T)=T^{\wedge}\left(\Phi_{M(A)}\right)$, it follows directly from the definition of $M_{00}(A)$ that also all elements of $M_{00}(A)$ have natural spectra.

Zafran $[\mathrm{Z}]$ was the first to investigate this concept. He worked in the context of group algebras $A=L^{1}(G)$ for locally compact abelian groups. Among other things, he observed that whenever $G$ is non-discrete there are multipliers in $M_{0}\left(L^{1}(G)\right)$ whose spectra are not natural. This, incidentally, tells us that the inclusion $M_{00}(A) \subset M_{0}(A)$ is indeed strict. In [LN1, Theorem 2.5(f)] it is then observed that whenever this inclusion is strict for a regular algebra $A$, then the two algebras $M_{00}(A)$ and $M_{0}(A)$ are very different: the former is regular and the latter is not.

Let us begin our discussion of natural local spectra by clarifying the relationship between this property and that of $T$ having natural spectrum. Since any operator $T$ with SVEP has the property that $\sigma(T)=\bigcup_{x \in X} \sigma_{T}(x)[\mathrm{V} 1]$, the spectrum of any multiplier $T$ on a commutative semisimple Banach algebra $A$ may be expressed as such a union. Clearly then, if $T$ has natural local spectra, $T$ will have natural spectrum. The converse is not true; more about this shortly, after we have recorded the following fact.

Theorem 9 [LN1, Proposition 2.1, ELN, Proposition 7]. If $T \in M(A)$ is decomposable then $T$ has natural spectrum. Moreover, if $A$ has approximate units then $T$ has natural local spectra. 
To what extent the last part of Theorem 9 holds without the added assumption on $A$ is not known.

Theorem 9 can of course be used to identify non-decomposable multipliers, and so we are in contact with the claim made after Theorem 4. A whole class of non-decomposable multipliers is identified this way in [ELN, Proposition 12]: for a compact abelian group $G$ consider any probability measure $\mu$ with independent powers. Assume that $\mu$ is symmetric, i.e. that $\mu(E)$ equals the complex conjugate of $\mu(-E)$ for every Borel set $E \in G$. Then it follows that $T_{\mu}$ does not have natural spectrum, hence is not decomposable. One way to see that $T_{\mu}$ does not have natural spectrum is via [GMcG, Theorem 6.1.1]: the spectrum of such $T_{\mu}$ is the entire closed unit disc, but the range of $T^{\wedge} \mid \Phi_{L^{1}(G)}$ will be real, by the symmetry of $\mu$.

The converses of the implications in Theorem 9 are both false. Concerning the first one, Zafran [Z] has an example of a multiplier which has natural spectrum without being decomposable: he specifies two multipliers $T_{1}$ and $T_{2}$ with natural spectra for which the sum $T_{1}+T_{2}$ does not have natural spectrum. We know, however, from Theorem 5 that if $A$ has a bounded approximate identity then the decomposable multipliers form an algebra. If both $T_{1}$ and $T_{2}$ were decomposable then so would be their sum, and since this is excluded by Theorem 9 , at least one of them must be non-decomposable. Some of the examples mentioned in the last paragraph exhibit these same features; they are explained in [ELN, Theorem 13]. Concerning the reversal of the second implication in Theorem 9 we want to say a little more.

Most work on natural local spectra has been done in the case when $A$ is assumed to be regular [ELN]. With this assumption and, additionally, that of assuming $A$ to be Tauberian, i.e. that the elements $a$ of $A$ for which $\operatorname{supp} a^{\wedge}$ is a compact subset of $\Phi_{A}$ form a dense ideal of $A$, some rather pleasing information is available. Indeed, if $A$ has these properties and also possesses approximate units (which do not have to form a bounded set) then it will always be the case for any $T \in M(A)$ that $Z_{T}(F)=A_{T}(F)^{-}$, for any closed $F \subseteq \mathbb{C}$ [ELN, Proposition 4]. Consequently, the minute we know that the $A_{T}(F)$-spaces are closed, we know that $T$ will have natural local spectra. The converse is clearly true, since the $Z_{T}(F)$-spaces are closed, so we have the following.

TheOREM 10 [ELN, Theorem 6]. If $A$ is a regular, Tauberian commutative semisimple Banach algebra with approximate units then $T \in M(A)$ has natural local spectra if and only if $A_{T}(F)$ is closed for every closed $F \subseteq \mathbb{C}$.

As usual, if we consider $T \in M_{0}(A)$, more is known.

THEOREM 11 [ELN, Corollary 10]. If $A$ is a regular, Tauberian commutative semisimple Banach algebra with approximate units, and if $T \in M_{0}(A)$ then $T$ has natural local spectra if and only if $T$ is decomposable. These properties hold precisely when $T \in M_{00}(A)$. 
Colin Graham has given examples (cf. [ELN]) of measures in $M_{0}\left(L^{1}(G)\right) \backslash$ $M_{00}\left(L^{1}(G)\right)$ with natural spectrum, for certain non-compact groups $G$, thus enabling us to distinguish the concept of natural spectrum from that of natural local spectra.

With the additional machinery available for group algebras, even more is known. Recall that a subset $K$ of a group $G$ is independent if for any choice of finitely many points $x_{i}$ from $K$ and integers $n_{i}$ the equation $n_{1} x_{1}+\ldots+n_{k} x_{k}=0$ implies that $n_{i} x_{i}=0$ for each $i=1, \ldots, k$.

TheOREM 12 [ELN, Theorem 13]. Let $K$ be a compact independent subset of a non-discrete locally compact abelian group $G$. Take any measure $\mu$ concentrated on $K$. Then $T_{\mu}: L^{1}(G) \rightarrow L^{1}(G)$ is decomposable precisely when $\mu$ is a discrete measure.

5. Multipliers with closed range. We conclude this survey with a brief description of an application of the characterization of spectral subspaces that we gave in Theorem 2. This application addresses itself to questions that arise from a spectacular result of abstract harmonic analysis, due to Host and Parreau [HP]. This result finally completed the answer to a question, originally asked by Hewitt, to which partial answers had been given by Glicksberg [G] and Ramsey and Wells [RW], among others. The Host-Parreau result says that a multiplier $T$ on the group algebra $L^{1}(G)$, where $G$ is a locally compact abelian group, will have closed range if and only if $T$ is the product of an idempotent multiplier and an invertible multiplier.

A similar factorization result for more general algebras, say any regular commutative semisimple Banach algebra, is not yet known. There are some conclusions available if the assumption of closed range for $T$ is replaced by that of closed range for $T^{2}$; loosely, the reason why the factorization result is known to hold in this case is that with information on $T^{2}$, the factorization is, so to speak, built into the situation from the outset [AL]. To avoid straying too far from local spectral theory only one result will be mentioned here, along with its technical basis. As the phrasing of Theorem 13 shows, we can make $T^{2}$ appear to "go away" in Theorem 14 only by imposing another restriction, that of injectivity.

TheOREM 13 [AL, Theorem 4.1]. Let $A$ be a commutative regular semisimple Banach algebra. If $T \in M(A)$, and if $T^{2}$ has closed range, then $T^{\wedge}$ is bounded away from zero on $\left\{\phi \in \Phi_{A} \mid T^{\wedge}(\phi) \neq 0\right\}$.

Sketch of proof. If $S:=T \mid(T A)^{-}$then $S$ is a multiplier on $B:=(T A)^{-}$ with closed range $T^{2}(A)$. Let $D_{\delta}$ denote the open disc of radius $\delta$, centered at 0 in the complex plane. As a consequence of the constancy result Theorem 2 , there is a $\delta_{0}>0$ for which, for every $0<\delta<\delta_{0}$, the analytic spectral subspaces $B_{S}\left(\mathbb{C} \backslash D_{\delta}\right)$ are all equal. Since $B_{S}\left(\mathbb{C} \backslash D_{\delta}\right)$ is an ideal with hull equal to $S^{\wedge^{-1}}\left(D_{\delta}\right)^{-}$, it is not hard to see that the equality of the spectral spaces forces $S^{\wedge^{-1}}\left(D_{\delta}\right)=$ 
$S^{\wedge^{-1}}(\{0\})=\emptyset$, the voidness resulting from regularity of $A$ and injectivity of $S$. But $S^{\wedge-1}\left(D_{\delta}\right)=\emptyset$ says that $S^{\wedge}$, and hence $T^{\wedge}$, is bounded away from zero on $\Phi_{B}=\left\{\phi \in \Phi_{A} \mid T^{\wedge}(\phi) \neq 0\right\}$.

Some standard facts about operators with closed range and about regular algebras then yield the following result which has been known for some time (see e.g. [DT, Theorem 2], also with another proof based on local spectral theory [ELN, Proposition 8]).

TheOREm 14 [AL, Theorem 4.3]. Let $T$ be a multiplier with closed range on a commutative Tauberian regular semisimple Banach algebra $A$. Then $T$ is injective if and only if $T$ is surjective.

So for multipliers with closed range on a regular commutative semisimple Tauberian Banach algebra there is a sort of Fredholm alternative: surjectivity of a multiplier is the same as invertibility. Thus, for instance, it is beyond the reach of multipliers in $M_{0}(A)$ to be surjective. And not just that: for any multiplier $T$ on a commutative regular semisimple Tauberian Banach algebra $A$ all points of the spectrum $\sigma(T)$ will be in the approximate point spectrum $\sigma_{\text {ap }}(T)$. This follows since if $\lambda \in \mathbb{C} \backslash \sigma_{\mathrm{ap}}(T)$ then $T-\lambda$ will be injective and have closed range.

\section{References}

[AL] P. Aiena and K. B. Laursen, Multipliers with closed range on regular commutative Banach algebras, Proc. Amer. Math. Soc., to appear.

[A1] E. Albrecht, Decomposable systems of operators in harmonic analysis, in: Toeplitz Centennial, Birkhäuser, Basel, 1982, 19-35.

[A2] - Spectral decompositions for systems of commuting operators, Proc. Roy. Irish Acad. 81A (1981), 81-98.

[Ap] C. Apostol, Decomposable multiplication operators, Rev. Roumaine Math. Pures Appl. 17 (1972), 323-333.

[BD] F. F. Bonsall and J. Duncan, Complete Normed Algebras, Springer, New York, 1973.

[CF] I. Colojoară and C. Foias, Theory of Generalized Spectral Operators, Gordon and Breach, New York, 1968.

[C] J. B. Conway, A Course in Functional Analysis, Springer, New York, 1985.

[DT] M. Dutta and U. B. Tewari, On multipliers of Segal algebras, Proc. Amer. Math. Soc. 72 (1978), 121-124.

[E] J. Eschmeier, Spectral decompositions and decomposable multipliers, Manuscripta Math. 51 (1985), 201-224.

[ELN] J. Eschmeier, K. B. Laursen and M. M. Neumann, Multipliers with natural local spectra on commutative Banach algebras, submitted.

[F] K.-H. Förster, Über die Invarianz einiger Räume, die zum Operator T- $\lambda$ A gehören, Arch. Math. (Basel) 17 (1966), 56-64.

[Fr] Şt. Frunză, A characterization of regular Banach algebras, Rev. Roumaine Math. Pures Appl. 18 (1973), 1057-1059.

[G] I. Glicksberg, When is $\mu * L_{1}$ closed?, Trans. Amer. Math. Soc. 160 (1971), 419-425. 
[GK] M. A. Gol'dman and S. N. Kračkovskiŭ, Invariance of certain spaces connected with the operator $A-\lambda I$, Soviet Math. Dokl. 5 (1964), 102-104.

[GMcG] C. C. Graham and O. C. McGehee, Essays in Commutative Harmonic Analysis, Springer, New York, 1979.

[H] H. Helson, Isomorphisms of abelian group algebras, Ark. Mat. 2 (1953), 475-487.

[HP] B. Host et F. Parreau, Sur un problème de I. Glicksberg: Les idéaux fermés de type fini de $M(G)$, Ann. Inst. Fourier (Grenoble) 28 (3) (1978), 143-164.

$[\mathrm{K}]$ T. Kato, Perturbation theory for nullity, deficiency and other quantities of linear operators, J. Analyse Math. 6 (1958), 261-322.

[L] R. Larsen, An Introduction to the Theory of Multipliers, Springer, New York, 1971.

[LN1] K. B. Laursen and M. M. Neumann, Decomposable multipliers and applications to harmonic analysis, Studia Math. 101 (1992), 193-214.

[LN2] - - - Local spectral theory and spectral inclusions, Glasgow Math. J., to appear.

[LV1] K. B. Laursen and P. Vrbová, Some remarks on the surjectivity spectrum of linear operators, Czechoslovak Math. J. 39 (1989), 730-739.

[LV2] - - - Intertwiners and automatic continuity, J. London Math. Soc. (2) 43 (1991), $149-155$.

[M] M. Mbekhta, Résolvant généralisé et théorie spectrale, J. Operator Theory 21 (1989), 69-105.

[MN] V. G. Miller and M. M. Neumann, Local spectral theory for multipliers and convolution operators, in: Proceedings of the Great Plains Operator Symposium at Iowa City, Birkhäuser, Basel, to appear.

[N] M. M. Neumann, Commutative Banach algebras and decomposable operators, Monatsh. Math. 113 (1992), 227-243.

[PV] V. Pták and P. Vrbová, Algebraic spectral subspaces, Czechoslovak Math. J. 38 (1988), 173-179.

[RW] L. T. Ramsey and B. B. Wells, Jr., Some results on the question: When is $\mu * L^{1}$ closed?, Indiana Univ. Math. J. 26 (1977), 987-996.

[S] C. Schmoeger, Ein Spektralabbildungssatz, Arch. Math. (Basel) 55 (1990), 484-489.

[SW] M. Ó Searcóid and T. T. West, Continuity of the generalized kernel and range of semi-Fredholm operators, Math. Proc. Cambridge Philos. Soc. 105 (1989), 513-522.

[V1] F.-H. Vasilescu, Residually decomposable operators in Banach spaces, Tôhoku Math. J. 21 (1969), 509-522.

[V2] - Analytic Functional Calculus, Reidel, Dordrecht, 1982.

[W] J. G. Wendel, On isometric isomorphism of group algebras, Pacific J. Math. 1 (1951), 305-311.

[Z] M. Zafran, On the spectra of multipliers, ibid. 47 (1973), 609-626. 